\title{
SulLa CORTESIA LINGUISTICA: UN'ANALISI SOCIOPRAGMATICA DI UN'INTERVISTA TRA Fabio Fazio e Antonio Di Pietro
}

EDOARDO NATALE*

ABSTRACT: Il presente articolo delinea un'analisi dell'atto linguistico del disaccordo, in una prospettiva sociopragmatica, con la finalità di stabilire delle differenze conversazionali e culturali. Si è privilegiato il disaccordo come tratto saliente perché permette di analizzare la qualità della cortesia linguistica presente all'interno di un'interazione avvenuta nel programma televisivo "Che tempo che fa" tra Fabio Fazio e il deputato Antonio Di Pietro. L'articolo è composto da una prima parte incentrata sulla ricognizione della nozione di faccia nella cortesia linguistica intesa come valido modello metodologico per l'indagine del fenomeno linguistico del disaccordo, mentre l'altra parte riguarda l'analisi del disaccordo segnalato all'interno della suddetta conversazione. L'analisi, condotta in base ai principi sociopragmatici di Spencer-Oatey, con l'utilizzo del modello della cortesia linguistica di Brown e Levinson, con gli innesti di Kerbrat-Orecchioni, all'interno di un approccio di matrice etnografico con i lavori di Duranti, mette in luce alcune diversità nella realizzazione del disaccordo tra i due parlanti analizzati in questo articolo. Parole chiave: sociopragmatica; disaccordo; cortesia linguistica.

RESUMO: O presente artigo esboça uma análise do ato linguístico da discordância, segundo uma perspectiva sociopragmática, com o objetivo

*Università di Modena e Reggio Emilia (UNIMORE) edona54@hotmail.com 
de estabelecer as diferenças conversacionais e culturais. Privilegiou-se a discordância como traço saliente porque ela permite analisar a qualidade da cortesia linguística presente numa interação ocorrida no programa televisivo "Che tempo che fa", entre Fabio Fazio e o deputado Antonio Di Pietro. O artigo é composto por uma primeira parte dedicada à explicação da noção de "face" na cortesia linguística, entendida como um modelo metodológico válido para a investigação do fenômeno linguístico da discordância; a segunda parte diz respeito à análise da discordância observada na conversa citada. Realizada com base nos princípios sociopragmáticos de Spencer-Oatey, utilizando o modelo da cortesia linguística de Brown e Levinson e as propostas de Kerbrat-Orecchioni, no âmbito de uma abordagem de cunho etnográfico inspirada nos trabalhos de Duranti, a análise destaca algumas diversidades na realização da discordância entre os dois falantes objeto deste artigo.

PALAVRAS-CHAVE: sociopragmática; discordância; cortesia linguística.

ABSTRACT: This paper outlines the linguistic analysis of the speech act of disagreement, in a sociopragmatic view, with the aim of establishing conversational and cultural differences. Disagreement was considered salient because it allows analyzing the quality of linguistic politeness in this interaction occurred in the Italian TV program "Che tempo che fa" between the interviewer Fabio Fazio and the deputy Antonio Di Pietro. The article consists in two parts: the first on the explanation of the notion of face in linguistic politeness intended as valid methodological model for the investigation of the linguistic phenomenon of disagreement; the second with the analysis of disagreement reported in the above conversation. The analysis, conducted according to the principles of sociopragmatics of Spencer-Oatey, and the model of linguistic politeness of Brown and Levinson, with the grafts of Kerbrat-Orecchioni, and an ethnographic approach based on the work of Duranti, highlights some differences in the implementation of disagreement between the two speakers of this article. KEY-WORDS: sociopragmatics; disagreement; politeness. 


\section{La visione della cortesia in Brown e Levinson}

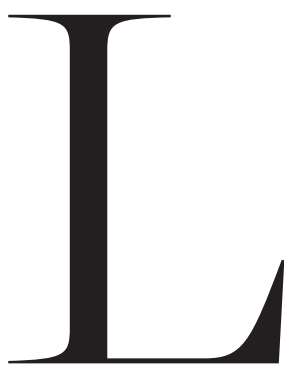

a visione di cortesia linguistica elaborata da Brown e Levinson (1978, 1987) riconosce un valore importante alla formulazione teorica di questo concetto, come sostenuto, ad esempio, in questo passaggio del libro Politeness. Some universals in usage:

[...] è al centro delle proposte di Grice, vale a dire che vi è un'ipotesi di lavoro elaborata dai conversazionalisti sulla natura razionale ed efficiente del parlare. $\grave{E}$ in opposizione a questa ipotesi che i modi cortesi del parlare appaiono come deviazioni, con la richiesta di spiegazioni razionali da parte del destinatario, al quale le ragioni della cortesia appaiono come apparente irrazionalità o inefficienza.

Per Brown e Levinson, una forte motivazione per cui non parlare in modo diretto, contravvenendo alle massime conversazionali di Grice, è da ricercare nel mantenimento della "faccia” intesa come immagine pubblica di sé (Goffman, 1967). Gli autori riconoscono, tuttavia, che la cortesia non è l'unica fonte di "deviazione" rispetto ai principi di cooperazione introdotti da Grice (1975): anche l'umorismo, il sarcasmo e l’ironia possono provocare effetti simili.

1 “[...] is at heart of Grice's proposals, namely that there is a working assumption by conversationalists of the rational and efficient nature of talk. It is against that assumption that polite ways of talking show up as deviations, requiring rational explanations on the part of the recipient, who finds in considerations of politeness reasons for the speaker's apparent irrationality or inefficiency." (1987: 4). Tranne nei casi in cui viene altrimenti specificato, le traduzioni di questo articolo sono dell'autore. 
Sostengono Brown e Levinson che la cortesia linguistica dovrebbe essere sempre comunicata e che la sua non comunicazione costituisce un messaggio che comporta, secondo la citata teoria griceana, un'implicatura conversazionale. Nel loro modello, la presenza o la mancanza di cortesia si spiega alla luce della cornice ideata da Goffman sul concetto di "faccia”, come costrutto universale elaborato culturalmente, come immagine pubblica che ogni membro di una società vuole affermare per se stesso (cf. 1955, pp. 213-231). Brown e Levinson caratterizzano la "faccia" come qualcosa che si può perdere, mantenere o rinforzare durante l'interazione e aggiungono che, dato il suo carattere di vulnerabilità sempre presente, i parlanti saranno impegnati a difenderla nel caso in cui dovesse essere minacciata. Visto il pessimismo indicato da Kerbrat-Orecchioni $(1992,1996)$, che sottende a ogni scambio conversazionale nell'approccio di Brown e Levinson, il presupposto è che sia meglio, per l'interesse di tutti, conservare la faccia dell'altro e agire in modo tale che gli altri ne siano consapevoli.

L'idea di fondo che governa la teoria della cortesia di Brown e Levinson è che alcuni atti linguistici sono di per sé minacciosi per la faccia (ad esempio, il disaccordo e la protesta) e, pertanto, devono essere mitigati (1987, p. 24). Saranno, tra gli altri, i lavori di Wierzbicka (1991) a mettere in luce come ogni gruppo linguistico sviluppi dei principi di cortesia dai quali derivano alcune strategie linguistiche che si rifanno alle norme comunicative presenti in un dato gruppo sociale.

Per Brown e Levinson è attraverso la conoscenza di queste strategie di cortesia di natura universale che il parlante ha successo nel comunicare il suo messaggio principale, dimostrando, in questo modo, la sua intenzione di essere cortese e, allo stesso tempo, di non voler perdere la faccia. Gli studiosi presentano atti linguistici che sono intrinsecamente minacciosi per la "faccia" del parlante, dell'ascoltatore o per entrambi: ad esempio, il disaccordo viene definito come un atto minaccioso per la faccia positiva dell'ascoltatore insieme ad altri atti linguistici come lamentarsi, criticare o parlare di argomenti tabù. Quando si è costretti a produrre questi atti linguistici che minacciano la faccia degli interagenti, il $f a-$ ce-work deve concentrarsi sulla riduzione della minaccia postulata da Brown e Levinson:

[...] abbiamo affermato che un agente portatore razionale di faccia tenderà a utilizzare strategie di minimizzazione dei FTA (Face Threatening Acts - atti 
minacciosi per la faccia), in base ad una valutazione razionale del rischio per la faccia dei partecipanti. Questo comportamento scaturisce da un ragionamento pratico, ossia inferendo le modalità migliori da utilizzare per soddisfare i fini dichiarati. ${ }^{2}$

Compiere un atto linguistico in modo diretto e senza atti di mitigazione è il modo più chiaro e inequivocabile di realizzare un atto di minaccia alla faccia, mentre le strategie di riparazione possono coinvolgere la cortesia positiva (mostrando solidarietà) oppure la cortesia negativa (mostrando distanza).

Brown e Levinson riprendono, inoltre, le affermazioni del modello di cortesia di Leech (1983) e la sua idea di varianti che potrebbero colpire un parlante tramite un FTA (Face Threatening Act, atto minaccioso per la faccia).

Queste varianti, indipendenti e sensibili al contesto culturale, giocano un ruolo importante nel modello di Brown e Levinson e sono le seguenti:

1. la distanza sociale (D) tra il parlante e l'ascoltatore, ovvero, il loro grado di familiarità e di solidarietà condivisa;

2. il potere relativo $(\mathrm{P})$ del parlante nei confronti dell'ascoltatore, nel senso della "forza persuasiva" del parlante sull'ascoltatore.

3. il grado d'imposizione (R) che vige in una determinata cultura, in termini di richiesta di beni e di servizi da parte dell'ascoltatore, ossia il diritto di realizzare alcuni atti e il grado di imposizione che l'ascoltatore può accettare.

Il modello teorico pensato da Brown e Levinson riconduce "il peso" (indicato come $\mathrm{W}$ nella formula) dei nostri atti linguistici in termini di "faccia" positiva o negativa durante la realizzazione di un FTA attraverso una formula, in cui S indica il parlante, $\mathrm{H}$ indica l'ascoltatore e R indica il grado d'imposizione da calcolare in questo modo:

$$
\mathrm{Wx}=\mathrm{D}(\mathrm{S}, \mathrm{H})+\mathrm{P}(\mathrm{H}, \mathrm{S})+\mathrm{Rx}
$$

Sarà il valore di Wx a determinare il grado di cortesia necessario a salvare la propria faccia ed è importante ricordare che queste varianti non devono essere viste come delle costanti tra gli individui. Durante l'interazione i partecipanti oscillano 
nella loro distanza sociale quando si trovano, ad esempio, in una situazione di lavoro oppure quando è presente un elevato grado di nervosismo. Allo stesso modo, il potere relativo cambia quando cambiano i ruoli e le responsabilità. La scelta di una data forma linguistica va quindi vista come una realizzazione specifica di una strategia di cortesia alla luce della valutazione del contesto della frase. Il modello esplicativo di Brown e Levinson può essere riassunto nei seguenti passaggi (1987, pp. 90-91):

- il parlante che intende compiere un FTA con la massima efficienza deve decidere se intende preservare la "faccia" del suo interlocutore, servendosi della cooperazione dell'interlocutore stesso che accetta il rischio derivante dalla partecipazione alla realizzazione dell'atto; oppure se pone in primo piano la conservazione della "faccia" dell'interlocutore;

- il parlante dovrebbe determinare il grado di minaccia alla faccia di un dato FTA e fino a che punto vuole minimizzare il rischio di perdita di faccia dovuto all'atto stesso, considerando fattori come il bisogno di chiarezza e quello di non sopravvalutare il grado potenziale di perdita della propria faccia;

- il parlante deve scegliere una strategia che provveda a "salvare la faccia": la presa in considerazione della cooperazione dell'interlocutore determina la strategia da scegliere per realizzare le aspettative dell'interlocutore stesso per quello specifico atto;

- il parlante deve allora scegliere un significato linguistico che lo soddisfi per la conclusione di quella strategia e considerare che ogni strategia abbraccia un'ampia scala di cortesia, nella quale sarà richiesto al parlante di considerare la specifica forma linguistica selezionata per coglierne gli effetti quando viene usata insieme ad altri elementi linguistici.

Un aspetto importante è capire che la scelta di una forma linguistica è determinata dalla responsabilità da parte del parlante verso il suo interlocutore durante l'interazione. Nell'ambito degli studi di pragmatica contrastiva appare necessario integrare la formula pensata da Brown e Levinson per definire in che modo si preserva o si crea "faccia" all'interno delle diverse culture. 


\section{Un caso di disaccordo: Fabio Fazio e Antonio Di Pietro}

Per dare un esempio di quanto abbiamo affermato finora, abbiamo selezionato l'intervista di Fabio Fazio a Antonio Di Pietro, realizzata durante il programma televisivo "Che tempo che fa", andato in onda su Rai 3, il 4 Aprile 2009, in prima serata, che rappresenta un classico esempio di interazione formalizzata ed istituzionalizzata (si veda Orletti, 1994 e 2000). Nell'intervista il controllo della gestione della parola da parte dell'intervistatore genera un rapporto di asimmetria capace di influenzare, come vedremo, molti aspetti dell'evento linguistico. L'intervistatore detiene teoricamente, in quest'intervista, il potere di influenzare l'interazione verbale non soltanto sul piano dello sviluppo sequenziale (decidendo la durata dell'intervista e dei turni di parola), ma anche sul piano delle tematiche, ovvero della formulazione di domande o dell'offerta di risposte.

Nell'analisi dell'intervista di Fabio Fazio a Antonio Di Pietro è utile considerare i principi sociopragmatici di Spencer-Oatey e Jiang (2003), che ci aiutano a classificare lo stile dell'intervistatore come distante, diretto, cordiale e modesto, e quello dell'intervistato come diretto, affermato e coinvolgente. Seguendo la terminologia di Spencer-Oatey e Jiang, possiamo dire che, in termini di costrizioni sociopragmatiche di diritti e doveri o compiti interazionali, Di Pietro esprime il diritto dei magistrati di poter cambiare ruolo professionale per trasformare la "persona" del magistrato in uomo politico, mentre per l'intervistatore Fabio Fazio questa scelta risulta più "costosa" che "benefica" (Spencer-Oatey, 2001) in termini di diritti per la persona del magistrato, all'interno del contesto culturale italiano che tende a delegittimare i magistrati che rivestono anche incarichi di tipo politico. Di fronte al disaccordo espresso da Fazio, Di Pietro evidenzia i benefici per la cittadinanza, intesa come "faccia collettiva" (Schwartz, 1992), ossia come gruppo che si identifica con una data realtà collettiva, rafforzata dalla presenza di magistrati nella sfera politica. L'intera durata del video può essere interpretata alla luce di un lungo disaccordo (Scott, 2002) tra i due protagonisti, dovuto a due stili conversazionali divergenti in termini sociopragmatici e alla loro diversa concezione della natura della "persona" del magistrato, disaccordo che si riflette anche sull'andamento stesso dell'intervista. 
Durante lo scambio verbale, in termini di massima di quantità (Grice, $1975)^{3}$ non viene ratificata tra i due parlanti una condivisione su come interpretare le intenzioni presenti nelle domande e nelle risposte date dall'intervistato e dall'intervistatore. Inoltre, questo lungo disaccordo sostenuto viene segnalato da parte degli interagenti non tanto sul contenuto offerto dall'intervistatore quanto sullo scopo delle domande che, secondo Di Pietro, servono a screditare il ruolo del magistrato che decide di fare politica. Ecco un passaggio della conversazione tra Fabio Fazio e Antonio Di Pietro in cui viene segnalata la presenza del disaccordo:

Fazio: $\quad$ però

Di Pietro: $\quad$ senta a me senta a me

Fazio: $\quad$ ma... non sono d'accordo con lei. Non sono affatto d'accordo ((gesto per indicare contrarietà))

Di Pietro: $\quad$ a lei.... preferisce mandarci i criminali?

Fazio: $\quad$ io //

Di Pietro: $\quad$ (??)

Fazio: $\quad$ (? ?) Aspetti, aspetti, mi faccia dire, però... scusi

Di Pietro: // a lei...... lo sa approfittiamo di questa trasmissione

Fazio: $\quad$ però non sono d'accordo con lei nel senso che considero il diritto [...]

Di Pietro: $\quad$ io mi sono dimesso prima di entrare in politica

Fazio: $\quad$ aveva detto che non sarebbe mai entrato in politica però lei

Di Pietro: $\quad$ e::: certo quando facevo il magistrato non sarei mai entrato

Fazio: [non subito dopo nel 97

Di Pietro: $\quad$ a 50 anni finito di aver fatto il magistrato dopo 2 anni che faccio il cittadino normale mi spiega qual è la ragione per chi non debba fare politica per permettere a tutti

3 Secondo l'intervistato Di Pietro, la massima di quantità di Grice ("Rendi il tuo contributo informativo quanto richiesto. Non rendere il tuo contributo più informativo di quanto richiesto.”) non viene rispettata da Fabio Fazio perché, secondo l'ospite, viene concesso troppo tempo a screditare l'operato dei magistrati, mentre poco tempo viene dedicato alla critica del malcostume di tanti politici italiani. 
Fazio: $\quad=/ /$ ma lei l'aveva detto non io

Di Pietro: $\quad$ l'ho detto in riferimento che facevo il magistrato

Fazio: $\quad$ ma

Di Pietro: [mi sono dimesso, per due anni ho fatto il magistrato. Ma si rende conto che una trasmissione come questa la sta dedicando al fatto che critica i magistrati che vanno a fare politica

Fazio: $\quad$ veramente no

Di Pietro: ma dedichi una bella trasmissione a criticare questi delinquentoni che fanno politica

Fazio: ((gesto per sottolineare la sua contrarietà)) ' veramente no, non è così e esattamente l'opposto' ((applauso del pubblico))

L’intervistatore usa la congiunzione avversativa "però” come elemento linguistico che fa da premessa al disaccordo nello scambio verbale tra il presentatore e l'intervistato. L'intervistato adopera, ripetendola due volte, la formula "senta a me", laddove il verbo "sentire" è utilizzato all'imperativo come marcatore discorsivo (Bazzanella, 1996) per indicare, in termini di forza illocutoria (Searle, 1969), una rimessa in discussione dell'affermazione precedente realizzata dall'intervistatore. Il raddoppiamento dello stesso enunciato rappresenta un rafforzativo della propria posizione e, allo stesso tempo, indica un disaccordo non rafforzato (Brown e Levinson, 1987) sull'enunciato precedente perché non sono accettati né il contenuto delle domande e né gli ends (Hymes, 1972) dell'intervista. L'interazione verbale dei due protagonisti dell'evento linguistico in esame prosegue con una rimessa in discussione da parte dell'intervistatore:

Fazio: $\quad$ ma... non sono d'accordo. Non sono affatto d'accordo.

In questo turno di parola (Duranti, 1997) di Fabio Fazio, il “ma” come congiunzione avversativa viene usato come elemento linguistico di "prefazione al disaccordo" (Caffi, 1999; Testa, 1988), seguito dal raddoppiamento dell'affermazione, che riprende in modo simile la mossa compiuta da Di Pietro. In questo turno di parola, 
il disaccordo viene segnalato in modo severo (Grimshaw, 1990) con l'atto linguistico "non sono d'accordo" e poi con l'avverbio di quantità "affatto" per marcare, con intensità ancora maggiore, il disaccordo in atto tra le domande poste da Fabio Fazio e l'interpretazione di Di Pietro. Nel turno di parola successivo, Fazio usa la congiunzione "però", facendola ricomparire come indicatore del disaccordo e, allo stesso tempo, mostrando il desiderio di riprendere il turno di parola per portare a termine il proprio pensiero su quello che sta accadendo all'interno dello scambio conversazionale (Duranti, 1997) tra il presentatore e l'ospite. Il disaccordo prosegue con il successivo turno di parola di Di Pietro, che inizia con l'imperativo di terza persona del verbo "scusare" ("scusi"), che in questo contesto ha la precipua funzione di esprimere disaccordo, dato che l'ospite continua a non accettare e a non sottoscrivere la visione di "persona" del magistrato conferita da Fazio. In seguito Di Pietro utilizza il raddoppiamento della formula "senta a me" come marcatore linguistico funzionale per cambiare il tema dell'argomentazione proposta dall'intervistatore e segnalare, di conseguenza, un prolungamento del disaccordo presente tra i due interagenti. Nel successivo turno di parola del presentatore compare la sequenza "scusi non sono d'accordo con lei nel senso che...”, con cui viene segnalato un disaccordo severo seguito da spiegazione (Scott, 2002), che ha la funzione di mitigare la minaccia per la cortesia positiva dell'intervistato. Questa scelta di esprimere in modo esplicito il proprio disaccordo può essere interpretata come bisogno di difendere la propria faccia in termini di cortesia positiva, dato che l'andamento dell'intervista mette in pericolo l'identità professionale del conduttore, confermando la produzione del disaccordo forte quando la propria identità è messa in pericolo (Rees-Miller, 2000).

Nel turno di parola di Antonio Di Pietro, costituito dai seguenti elementi linguistici "lei sta dedicando una trasmissione come questa nel criticare i magistrati che decidono di fare politica", si vede che l'intervistato Di Pietro reagisce alla spiegazione offerta da Fazio, basando la sua argomentazione sulla massima di quantità di Grice (1975): Di Pietro giudica eccessivo il tempo adoperato per parlare della partecipazione alla vita politica di alcuni magistrati, tempo che andrebbe invece dedicato, seguendo la stessa massima della quantità, a criticare i tanti "delinquenti" che siedono nel parlamento italiano.

Nella replica di Fazio che dice "veramente no, non è così è esattamente l'opposto", l'intervistatore ribadisce la propria contrarietà segnalando il disaccordo in 
modo ancora più forte e negando il cultural script ${ }^{4}$ offerto da Di Pietro, vale a dire, esplicitando che il suo è contrario a quello fornito da Di Pietro.

L’uso dell'avverbio "veramente" possiede la funzione di segnalare la propria verità; l'enunciato "non è così", con l'avverbio di negazione e l'avverbio di modo, indica il rifiuto dell'analisi fatta da Di Pietro, così come l'avverbio "esattamente” è di nuovo un elemento che tende ad indicare la verità e la precisione delle proprie affermazioni. Infine, l'aggettivo "opposto" viene utilizzato per segnalare il proprio disaccordo di fronte alle minacce di Di Pietro in termini di cortesia positiva.

Per uscire da questa situazione, l'intervistatore cambierà argomento introducendo una nuova domanda come elemento che sancisce la fine del disaccordo incentrato su un tema preciso della conversazione. Di fatto, la risoluzione del conflitto interazionale tra i due interagenti si risolve con un disaccordo tralasciato (Vulchinich, 1990), che porta al cambiamento di argomento per proseguire l'intervista come forma di riparazione della faccia positiva di Fabio Fazio.

Di fatto, emergerà una visione contrapposta della "persona" del magistrato che, da Fazio, è intesa come principio divino, mentre Di Pietro difende la visione di una giustizia dal volto umano, in cui solo nella legalità le persone possono vivere una condizione di sviluppo sociale ed economico.

Vediamo ora l'interazione in cui si tralascia il disaccordo severo (Grimshaw, 1990) tra i due interlocutori, segnato dall'imbarazzo di Fazio e dalla comunicazione non verbale proveniente dallo sguardo severo di Di Pietro, per cambiare argomento e parlare di Mario Chiesa:

Fazio: $\quad$ parliamo ancora di magistrati, è obbligatorio il revival?

Di Pietro: $\quad$ come?

Fazio: dico è obbligatorio il revival? Mario Chiesa da cui tutto era cominciato. Di nuovo, ma è.

Di Pietro: $\quad$ guarda, grazie davvero per la domanda. Non tanto per la situazione di Chiesa, ma

Fazio: $\quad$ questo che vuol dire?

Anche se l'intervistatore intende continuare a parlare di magistratura, come indica nella prefazione alla sua domanda, la domanda stessa possiede un elemento

4 La nozione di cultural script di Wierzbicka (1991) e ripresa da Béal (1993) rappresenta un modo originale di tradurre le intenzioni attribuibili a Di Pietro, in quanto il cultural script di Di Pietro potrebbe essere sintetizzato in questo modo: i magistrati hanno diritto di fare politica; quelli che si oppongono a questo fatto sono complici dei politici; quindi non sei degno di meritare il mio rispetto e di quello della gente normale. Il cultural script di Fabio Fazio potrebbe essere ripreso in questi termini: voglio parlare di questo tema in un altro modo; so che questo tema è complicato da vedere in modo neutro; ma non voglio essere considerato come complice del potere politico. 
linguistico, la parola "revival", che ha la funzione pragmatica di alleggerire il contenuto della domanda, tramite l'utilizzo di un repertorio linguistico che "indessicalizza" (Duranti, 2007) la conversazione in maniera differente, creando un cambiamento di prosodia in termini intonativis. Pare, infatti, che il termine "revival" in un primo momento non venga capito da Di Pietro e ciò provoca il rapido intervento di Fazio che, ripetendo la domanda per aumentarne i benefici tramite un'ulteriore esplicitazione, ha lo scopo di agevolare la risposta di Di Pietro. Questa domanda offre inoltre una possibilità per la faccia dell'ospite: quella di mettere in rilievo la faccia positiva del suo intervistatore tramite l'enunciato "grazie davvero per la domanda", il quale permette di ritornare ad un equilibrio interazionale tra i due interagenti, in termini sia di costi/benefici, ma soprattutto in termini di diritti/doveri che competono alla persona dell'ospite, all'interno di un'interazione verbale compiuta in uno studio televisivo. Quest'offerta di faccia positiva, espressa con la formula "la ringrazio per la domanda", potrebbe sancire anche un gesto di riconoscimento in termini di cortesia positiva verso l'intervistatore per aver individuato una domanda interessante, secondo l'opinione dell'ospite.

Nel comportamento linguistico ed extralinguistico di Di Pietro è, inoltre, interessante evidenziare una preoccupazione per la presenza degli spettatori in studio, che manifesta indirizzando gli occhi verso di loro, come se volesse interpretare il loro umore e la loro vita quotidiana intesi come faccia collettiva (Schwartz, 1992). Quest'atteggiamento dimostra un'attenzione verso il reale destinatario di questa interazione, ossia il pubblico in studio e soprattutto quello a casa.

Un altro disaccordo viene rappresentato dalla seguente sequenza di conversazione (Duranti, 1997):

Fazio:

è interessante la sua analisi per capire come mai un uomo d'ordine, dopo la sua carriera, la sua vicenda professionale precedente, non vada più a dare fastidio, a togliere voti al centro destra ma al centro sinistra?

Di Pietro: $\quad$ E chi l'ha detto? E quello sto facendo. Io le sto dicendo. Elettori del centro destra, guardate, non è che votando quel governo state meglio di prima ma è come prima e peggio di prima

5 La prosodia rappresenta un mezzo linguistico molto utile per segnalare le proprie emozioni durante l'interazione verbale, come viene sottolineato in Bettoni (2006:19) attraverso lo studio dell'intonazione, del ritmo, della durata e dell'accento nel linguaggio parlato. 
In quest'esempio vediamo la presentazione di una realtà tramite una riformulazione del presentatore, in cui si propone l'azione dell'ospite Di Pietro come, di fatto, un'azione che va a togliere dei voti al centro-sinistra, essendo suggerita da Fabio Fazio un'implicatura conversazionale di questa natura, vale a dire che un uomo di giustizia dovrebbe attingere i suoi voti soprattutto nell'elettorato politico del centro-destra. Possiamo vedere nell'intervento dell'intervistatore un tentativo di indicare l'azione politica di Di Pietro come un'azione benefica soprattutto per il centro-destra poiché, di fatto, indebolisce il centro-sinistra, facendo pagare un costo al proprio alleato.

La risposta di Di Pietro, davanti ad una riformulazione percepita come un attacco alla sua faccia in termini di cortesia negativa, avviene con la formula "E chi l'ha detto", la quale rappresenta una sfida e un totale capovolgimento della presentazione dei fatti, in cui la presenza della congiunzione coordinativa copulativa "e" descrive un modo per riagganciarsi al contesto (Goodwin e Duranti, 1992) precedente in termini di “challenge” (Grimshaw, 1990) al contenuto presentato dall'intervistatore. Quindi l'ospite esprime il suo dovere di difendere i benefici della sua azione, compiendo, di fatto, un'azione che sancisce dei costi per la faccia collettiva (Schwartz, 1992) del centro-sinistra, rivolgendo una richiesta in diretta all'elettorato di destra con uno stile di tipo affermato ${ }^{6} \mathrm{e}$, si può aggiungere, un po’ ironicocomico. Quest'azione, condotta senza tenere conto che il pubblico complessivo del programma di Fazio tende ad un orientamento politico prevalentemente di centrosinistra, fa scaturire un effetto di paradosso che provoca l'ilarità del pubblico in studio.

Anche se l’intervista ha avuto molti momenti di conflittualità evidenziati dalla presenza di elementi di disaccordi precedentemente citati, l'ospite Di Pietro non perde di vista la necessità di riequilibrare il rapporto interpersonale tramite forme di risposte che si rifanno ad uno stile coinvolgente e/o caloroso (Spencer-Oatey e Jiang, 2003), come nel seguente frammento:

Fazio: Le devo porre tre domande e la prego di assecondarmi nelle risposte?

Di Pietro: $\quad$ (pausa) nel senso che devo dire di sì?

Fazio: $\quad$ No nel senso che deve cortesemente rispondere nel modo breve.

6 Vedi Spencer-Oatey e Jiang (2003) a proposito delle costrizioni sociopragmatiche come indicatori per analizzare lo stile interazionale del parlante. In questo lavoro il termine stile interazionale viene adoperato in modo sinonimico rispetto al termine "ethos". 
In questo scambio vediamo la domanda di Fazio come apparente tentativo di cordialità verso l'ospite Di Pietro, comunicata attraverso un compito da svolgere da parte dell'ospite. Questo tentativo di coinvolgimento dell'intervistatore viene colto da Di Pietro tramite una risposta data in tono scherzoso e caloroso. Questa risposta non troverà, però, un riscontro caloroso da parte di Fazio, il quale cerca di ristabilire una certa distanza sociale e freddezza interazionale per segnalare che la guida dell'intervista è nelle sue mani e che, pertanto, occorre rispettare il dovere/compito dell'intervistatore (cortesia negativa).

Appare in questa prossima sequenza conversazionale (Duranti, 1997) un passaggio singolare dell'intervista tra Fabio Fazio e Antonio Di Pietro, nel quale emerge con chiarezza la nozione di persona quando, durante l'intervista, riscontriamo questo turno di parola da parte di Di Pietro:

Di Pietro: $\quad$ torno a ripetere...e allora mi lasci fare un attimo il Fazio della situazione. Lei un condannato con sentenza penale passata in giudicato lo ricandiderebbe o no?

Fazio: $\quad$ io::: no

Di Pietro: $\quad$ e diglielo a Berlusconi

In questo turno di parola, vediamo come l'intervistato cambia la nozione di persona (Duranti, 2007), assumendo il ruolo di intervistatore ("mi lasci fare un attimo il Fazio della situazione”), in modo tale da poter configurare al meglio la sua intenzione di mettere in risalto il diritto di criticare il malcostume di numerosi dirigenti politici italiani e allo scopo di ottenere dei benefici in termini di faccia positiva da parte del pubblico in studio e a casa. Questa mossa comunicativa conferma la riflessione di Orletti (1994) sul ruolo asimmetrico e di potere conferito alla persona che gestisce l'intervista.

\section{Bibliografia}

BAZZANELLA, Carla. "I segnali discorsivi”. In: Grande grammatica italiana di consultazione, L. Renzi, G. Salvi, A. Cardinaletti (a cura di), Bologna, Il Mulino, 1996. 
BÉAL, Christine. Les stratégies conversationnelles en français et en anglais. Conventions ou reflet de divergences culturelles profondes? Langue française 98, 1993, pp. 79-106.

BETTONI, Camilla. Usare un'altra lingua. Guida alla pragmatica interculturale, Laterza Editori, Roma-Bari, 2006.

BROWN, Penelope \& Levinson Stephen. "Universals in language use: Politeness phenomena”. In: E. Goody (ed.), Questions and politeness. Strategies in social interaction. Cambridge, CUP, 1978, pp. 56-289.

BROWN, Penelope \& Levinson, Stephen. Politeness: Some universals in language usage, Cambridge, Cambridge University Press, 1987.

CAFFI, Claudia. On Mitigation. Journal of Pragmatics 31, 1999, pp. 881-909.

DURANTI, Alessandro. Linguistic Anthropology, Cambridge, Cambridge University Press, 1997 (tradotto in italiano con il titolo Antropologia del linguaggio, Roma, Meltemi, 2005).

DURANTI, Alessandro. Etnopragmatica. La forza nel parlare, Roma, Carocci Editore, 2007.

GOFFMAN, Erving. “On Face-work: An Analysis of Ritual Elements of Social Interaction.” Psychiatry: Journal for the Study of Interpersonal Processes 18 (3), 1995, pp. 213-231.

GOFFMAN, Erving. Interactional rituals. Essays on the face-to-face behavior, New York, Doubleday, 1967.

GOODWIN, Charles \& Duranti, Alessandro. "Rethinking context: An introduction”. In: C. Goodwin; A. Duranti (eds.), Rethinking Context: Language as an Interactive Phenomenon, Cambridge, Cambridge University Press, 1992, pp. 1-42.

GRICE, Paul. "Logic and conversation”. In: D. Davidson; G. Harman (eds.). The logic of grammar, Encino/California, Dickenson, 1975, pp. 64-74 (trad. it. Paul Grice, Logica e conversazione, Bologna, il Mulino, 1993).

GRIMSHAW, Allen. Conflict Talk. Cambridge, Cambridge University Press, 1990.

HYMES, Dell. "Models of the interaction of language and social life". In: J.J. Gumperz; D. Hymes (eds.). Directions in sociolinguistics. The ethnography of communication, New York-Chicago, Holt, Rinehart and Winston, 1972, pp. 35-71.

KERBRAT-Orecchioni, Catherine. Les interactions verbales, tomo 2, Paris, Armand Colin, 1992.

KERBRAT-Orecchioni, Catherine. La conversation, Paris, Seuil («Mémo»), 1996.

LEECH, Geoffrey. The principles of pragmatics, London, Longman, 1983.

ORLETTI, Franca (a cura di). Fra conversazione e discorso. L'analisi dell'interazione verbale, Roma, La Nuova Italia Scientifica, 1994.

ORLETTI, Franca. La conversazione diseguale. Potere e interazione, Roma, Carocci, 2000.

REES-Miller, Janie. Power, severity, and context in disagreement. Journal of Pragmatics 32, 2000, pp. 1087-1111.

SCHWARTZ, Shalom. Universals in the content and structure of values: theoretical advances and empiricals tests in 20 countries. Advances in Experimental Social Psychology 25, 1992, pp.1-65.

SCOTT, Suzanne. Linguistic Feature Variation within Disagreement: an Empirical Investigation, Text 22 (2), 2002, pp. 301-328.

SEARLE, John. Speech acts: an essay in the philosophy of language, London, Cambridge University Press, 1969. 
SPENCER-OATEY, Helen. Culture and communication: Cross-cultural Explorations of the Sociocultural Interactional Principles, Paper presented at IRIC Conference 'Comparing cultures', 2001.

SPENCER-Oatey, Helen \& Jiang, Wenying. Explaining cross-cultural pragmatic findings: Moving from politeness maxims to sociopragmatic interactional principles (SIPs). Journal of Pragmatics 35, 2003, pp. 1633-1650.

TESTA, Renata. Interruptive strategies in English and Italian conversation: smooth versus contrastive linguistic preferences. Multilingua 7, 1988, pp. 285-312.

VUCHINICH, Samuel. "The sequention organization of closing in verbal family conflict". In: A. D. Grimshaw (ed.) Conflict Talk: Sociolinguistic Investigations of Arguments in Conversations, New York, Cambridge University Press, 1990, pp. 118-138.

WIERZBICKA, Anna. Cross-cultural pragmatics: the semantics of human interaction, Berlin-New York, Mouton de Gruyter, 1991. 